\title{
THE VERTEX DISTANCE COMPLEMENT SPECTRUM OF SUBDIVISION VERTEX JOIN AND SUBDIVISION EDGE JOIN OF TWO REGULAR GRAPHS
}

\author{
Ann Susa Thomas \\ Department of Mathematics, St Thomas College, \\ Kozhencherry-689641, Kerala, India \\ anns11thomas@gmail.com \\ Sunny Joseph Kalayathankal \\ Jyothi Engineering College, \\ Cheruthuruthy, Thrissur-679531, Kerala, India \\ sjkalayathankal@jecc.ac.in \\ Joseph Varghese Kureethara \\ Department of Mathematics, Christ University, \\ Bangalore-560029, Karnataka, India \\ frjoseph@christuniversity.in
}

\begin{abstract}
The vertex distance complement (VDC) matrix $C$, of a connected graph $G$ with vertex set consisting of $n$ vertices, is a real symmetric matrix $\left[c_{i j}\right]$ that takes the value $n-d_{i j}$ where $d_{i j}$ is the distance between the vertices $v_{i}$ and $v_{j}$ of $G$ for $i \neq j$ and 0 otherwise. The vertex distance complement spectrum of the subdivision vertex join, $G_{1} \dot{\bigvee} G_{2}$ and the subdivision edge join $G_{1} \bigvee G_{2}$ of regular graphs $G_{1}$ and $G_{2}$ in terms of the adjacency spectrum are determined in this paper.
\end{abstract}

Keywords: Distance matrix, Vertex distance complement spectrum, Subdivision vertex join, Subdivision edge join.

\section{Introduction}

Spectral graph theory deals with the study of the eigenvalues of various matrices associated with graphs. Initially, the spectrum of the adjacency matrix of a graph was studied. Collatz and Sinogowitz initiated the exploration of this topic in 1957 [2]. Since then spectral theory of graphs is an active research area $[1,3]$.

In this paper, we consider the matrix derived from a type of distance matrix, viz., vertex distance complement (VDC) matrix. The VDC spectra of some classes of graphs are found in [8, 9]. The VDC matrix $C$ of a graph $G[7]$ is defined as follows

$$
C= \begin{cases}n-d_{i j}, & i \neq j \\ 0, & i=j,\end{cases}
$$

where $d_{i j}$ is the distance between the vertices $v_{i}$ and $v_{j}$ of $G$ and $n$ denotes the number of vertices of $G$. 
The subdivision graph $S(G)$ of a graph $G$ is obtained by inserting a new vertex of degree two in every edge of $G$. Let $V(G)$ and $I(G)$ denote respectively the existing vertex set and the set of the newly introduced vertices of the subdivision graph $S(G)$ of a graph $G$. The adjacency spectrum of two joins, $G_{1} \dot{\bigvee} G_{2}$ and $G_{1} \bigvee G_{2}$, based on subdivision graph was determined in [4]. The distance spectrum of the same was calculated in [6].

Throughout this article we consider connected simple graphs of diameter at most two. We determine the VDC spectrum of $G_{1} \dot{\bigvee} G_{2}$ and $G_{1} \bigvee G_{2}$ when $G_{1}$ and $G_{2}$ are regular graphs. The eigenvalues of $V D C(G)$ are called the $V D C$-eigenvalues of $G$ and they form the $V D C$ spectrum of $G$, denoted by $\operatorname{spec}_{V D C}(G)$. We denote $J$ and $I$ as the all-one matrix and identity matrix, respectively, of appropriate orders.

The definitions of the subdivision graphs are as follows.

Definition 1 [4]. The subdivision-vertex join $G_{1} \dot{\vee} G_{2}$ of two vertex disjoint graphs $G_{1}$ and $G_{2}$ is the graph obtained from $S\left(G_{1}\right)$ and $G_{2}$ by joining each vertex of $V\left(G_{1}\right)$ with every vertex of $V\left(G_{2}\right)$.

Definition 2 [4]. The subdivision-edge join $G_{1} \bigvee G_{2}$ of two vertex disjoint graphs $G_{1}$ and $G_{2}$ is the graph obtained from $S\left(G_{1}\right)$ and $G_{2}$ by joining each vertex of $I\left(G_{1}\right)$ with every vertex of $V\left(G_{2}\right)$.

The following results are very useful for computing the VDC spectrum.

Lemma 1 [3]. Let $G$ be an $r$-regular graph with adjacency matrix $A$ and incidence matrix $R$. Let $A(L(G))$ denote the adjacency matrix of the line graph $L(G)$ of $G$. Then,

$$
R R^{T}=A+r I, \quad R^{T} R=A(L(G))+2 I .
$$

Also,

$$
J R=2 J=R^{T} J, \quad J R^{T}=r J=R J .
$$

Lemma 2 [3]. Let $G$ be r-regular $(n ; m)$ graph with $\operatorname{spec}(G)=\left\{\lambda_{1}, \lambda_{2}, \cdots, \lambda_{n}\right\}$. Then

$$
\operatorname{spec}(L(G))= \begin{cases}2 r-2, & \\ \lambda_{i}+r-2, & i=2,3, \ldots, n, \\ -2, & m-n \text { times }\end{cases}
$$

Also, $Z$ is an eigenvector corresponding to the eigenvalue - 2 if and only if $R Z=0$ where $R$ is the incidence matrix of $G$.

Theorem 1 (Perron-Frobenius). If all entries of an $n \times n$ matrix are positive, then it has a unique maximal eigenvalue. Its eigenvector has positive entries.

\section{The VDC spectrum of $G_{1} \dot{\bigvee} G_{2}$}

Theorem 2. Let $G_{i}$ be an $r_{i}$ regular graph with $n_{i}$ vertices and $m_{i}$ edges, for $i=1,2$. If $\left\{\lambda_{i 1}, \lambda_{i 2}, \ldots, \lambda_{i n_{i}}\right\}$ denotes the adjacency spectrum corresponding to the adjacency matrix $A_{i}$ of $G_{i}$, the $\operatorname{spec}_{V D C}\left(G_{1} \dot{\bigvee} G_{2}\right)$ consists of

(i) $2 \lambda_{1 i}+2 r_{1}-n+2$, for $i=2,3, \ldots, n_{1}$; 
(ii) $-n$, repeated $m_{1}-1$ times;

(iii) $\lambda_{2 i}-n+2$, for $i=2,3, \ldots, n_{2}$;

(iv) the 3 roots of the equation

$$
\begin{gathered}
x^{3}-\left(n_{1} n-2 n_{1}+n_{2} n-2 n_{2}+m_{1} n^{2}-4 m_{1}+4 r_{1}+r_{2}-3 n+4\right) x^{2} \\
-\left(2 n_{1} n_{2} n-3 n_{1} n_{2}+n_{1} m_{1}-2 n_{1} r_{1} n+2 n_{1} r_{1}-n_{1} r_{2} n+2 n_{1} r_{2}+2 n_{1} n^{2}\right. \\
-6 n_{1} n+4 n_{1}+2 n_{2} m_{1} n-4 n_{2} m_{1}-4 n_{2} r_{1} n+8 n_{2} r_{1}+2 n_{2} n^{2}-6 n_{2} n+4 n_{2}-m_{1} r_{2} n \\
\left.+4 m_{1} r_{2}+2 m_{1} n^{2}-8 m_{1} n+4 m_{1}-4 r_{1} r_{2}+8 r_{1} n-8 r_{1}+2 r_{2} n-2 r_{2}-3 n^{2}+8 n-4\right) x \\
-\left(2 n_{1} n_{2} m_{1}-4 n_{1} n_{2} r_{1} n+4 n_{1} n_{2} r_{1}+2 n_{1} n_{2} n^{2}-3 n_{1} n_{2} n-n_{1} m_{1} r_{2}\right. \\
+n_{1} m_{1} n-2 n_{1} m_{1}+2 n_{1} r_{1} r_{2} n-2 n_{1} r_{1} r_{2}-2 n_{1} r_{1} n^{2}+6 n_{1} r_{1} n-4 n_{1} r_{1}-n_{1} r_{2} n^{2} \\
+2 n_{1} r_{2} n+n_{1} n^{3}-4 n_{1} n^{2}+4 n_{1} n+2 n_{2} m_{1} n^{2}-8 n_{2} m_{1}-4 n_{2} r_{1} n^{2}+8 n_{2} r_{1} n+n_{2} n^{3} \\
-4 n_{2} n^{2}+4 n_{2} n-m_{1} r_{2} n^{2}+2 m_{1} r_{2} n+4 m_{1} r_{2}+m_{1} n^{3}-4 m_{1} n^{2}+8 m_{1}-4 r_{1} r_{2} n \\
\left.+4 r_{1} n^{2}-8 r_{1} n+r_{2} n^{2}-2 r_{2} n-n^{3}+4 n^{2}-4 n\right)=0
\end{gathered}
$$

where $n=n_{1}+m_{1}+n_{2}$.

P r o o f. Given that $G_{1}$ and $G_{2}$ are regular graphs with regularity $r_{1}$ and $r_{2}$ respectively. Let $R$ be the incidence matrix of $G_{1}$ and $A\left(L\left(G_{1}\right)\right.$ be the adjacency matrix of the line graph of $G_{1}$. The distance matrix of a graph with diameter at most two and adjacency matrix $A$ can be rewritten as $A+2 \bar{A}$ or $2(J-I)-A[5]$.

The subdivision-vertex join $G_{1} \dot{\bigvee} G_{2}$ has $n=n_{1}+m_{1}+n_{2}$ vertices. With the proper labeling of vertices, the VDC matrix of $G_{1} \dot{V} G_{2}$ is a square matrix of order $n$ given by

$$
C=\left(\begin{array}{ccc}
(n-2)(J-I) & (n-3) J+2 R & (n-1) J \\
(n-3) J+2 R^{T} & (n-4)(J-I)+2 A\left(L\left(G_{1}\right)\right) & (n-2) J \\
(n-1) J & (n-2) J & (n-2)(J-I)+A_{2}
\end{array}\right)
$$

Let $X$ be an eigenvector corresponding to the eigenvalue $\lambda_{1 i} \neq r_{1}$ of $A_{1}$. Using Lemma 1, we note that

$$
A\left(L\left(G_{1}\right)\right) R^{T} X=\left(\lambda_{1 i}+r_{1}-2\right) R^{T} X .
$$

Hence, $\lambda_{1 i}+r_{1}-2$ are the eigenvalues of $A\left(L\left(G_{1}\right)\right)$ with an eigenvector $R^{T} X$.

By Perron-Frobenius theorem, $X$ and $R^{T} X$ are orthogonal to the all-one vector $J$.

Let

$$
\Upsilon=\left(\begin{array}{c}
X \\
R^{T} X \\
0
\end{array}\right)
$$

Then,

$$
2 \lambda_{1 i}+2 r_{1}-n+2, \quad i=2,3, \ldots, n_{1}
$$


is an eigenvalue of the VDC matrix of $G_{1} \dot{\bigvee} G_{2}$ corresponding to the eigenvector $\Upsilon$. This is because

$$
\begin{gathered}
\left(\begin{array}{ccc}
(n-2)(J-I) & (n-3) J+2 R & (n-1) J \\
(n-3) J+2 R^{T} & (n-4)(J-I)+2 A(L(G)) & (n-2) J \\
(n-1) J & (n-2) J & (n-2)(J-I)+A_{2}
\end{array}\right)\left(\begin{array}{c}
X \\
R^{T} X \\
0
\end{array}\right) \\
=\left(\begin{array}{c}
\left.-(n-2) X+2\left(A_{1}+r_{1} I\right)\right) X \\
2 R^{T} X-(n-4) R^{T} X+2 A\left(L\left(G_{1}\right)\right) R^{T} X \\
0
\end{array}\right)=\left(\begin{array}{c}
\left(2 \lambda_{1 i}+2 r_{1}-n+2\right) X \\
\left(2 \lambda_{1 i}+2 r_{1}-n+2\right) R^{T} X \\
0
\end{array}\right) \\
=\left(2 \lambda_{1 i}+2 r_{1}-n+2\right)\left(\begin{array}{c}
X \\
R^{T} X \\
0
\end{array}\right) .
\end{gathered}
$$

By a similar reasoning, if $Y$ is an eigenvector of $A\left(L\left(G_{1}\right)\right)$ corresponding to the eigenvalue $\lambda_{1 i}+r_{1}-2$, for $i=2,3, \ldots, n_{1}$,

$$
\Phi=\left(\begin{array}{c}
R Y \\
-Y \\
0
\end{array}\right)
$$

is an eigenvector of VDC matrix of $G_{1} \dot{\vee} G_{2}$ corresponding to the eigenvalue $-n$. (Note that the line graph of a regular graph is also regular).

Hence, $-n$ is an eigenvalue of $G_{1} \dot{\bigvee} G_{2}$ repeated $n_{1}-1$ times.

Now, -2 is an eigenvalue of $A\left(L\left(G_{1}\right)\right)$ with multiplicity $m_{1}-n_{1}$. Let $\mathrm{Z}$ be an eigenvector of $A\left(L\left(G_{1}\right)\right)$ corresponding to the eigenvalue -2 . Then, by Lemma $2, R Z=0$ and by PerronFrobenius theorem, $J Z=0$.

Let

$$
\Omega=\left(\begin{array}{l}
0 \\
Z \\
0
\end{array}\right) .
$$

Then $-n$ is an eigenvalue of the VDC matrix of $G_{1} \dot{V} G_{2}$ repeated $m_{1}-n_{1}$ times with an eigenvector $\Omega$. This is because

$$
\begin{aligned}
& \left(\begin{array}{ccc}
(n-2)(J-I) & (n-3) J+2 R & (n-1) J \\
(n-3) J+2 R^{T} & (n-4)(J-I)+2 A(L(G)) & (n-2) J \\
(n-1) J & (n-2) J & (n-2)(J-I)+A_{2}
\end{array}\right)\left(\begin{array}{l}
0 \\
Z \\
0
\end{array}\right) \\
& =\left(\begin{array}{c}
0 \\
-(n-4) Z+2 A\left(L\left(G_{1}\right)\right) Z \\
0
\end{array}\right)=\left(\begin{array}{c}
0 \\
-n Z \\
0
\end{array}\right) .
\end{aligned}
$$

In total, $-n$ is an eigenvalue of $G_{1} \dot{\vee} G_{2}$ repeated $m_{1}-1$ times.

Now, let $\lambda_{2 i} \neq r_{2}$ be an eigenvalue of $G_{2}$ with an eigenvector W. Since $G_{2}$ is regular, $J W=0$. Hence

$$
\Psi=\left(\begin{array}{c}
0 \\
0 \\
W
\end{array}\right)
$$

is an eigenvector of the VDC matrix of $G_{1} \dot{\bigvee} G_{2}$ corresponding to the eigenvalue $\lambda_{2 i}-n+2$, for $i=2,3, \ldots n_{2}$. Thus, we have obtained $n_{1}+m_{1}+n_{2}-3$ eigenvalues.

The remaining three eigenvalues are to be determined. We note that all the eigenvectors constructed so far, are orthogonal to

$$
\left(\begin{array}{l}
J \\
0 \\
0
\end{array}\right), \quad\left(\begin{array}{l}
0 \\
J \\
0
\end{array}\right) \text { and }\left(\begin{array}{l}
0 \\
0 \\
J
\end{array}\right) .
$$


The remaining three eigenvectors are spanned by these three vectors and is of the form

$$
\Theta=\left(\begin{array}{c}
\alpha J \\
\beta J \\
\gamma J
\end{array}\right)
$$

for some $(\alpha, \beta, \gamma) \neq(0,0,0)$.

Thus, if $\rho$ is an eigenvalue of the VDC matrix with an eigenvector $\Theta$, then from $C \Theta=\rho \Theta$, we can see that the remaining three eigenvalues are obtained from the matrix

$$
\left(\begin{array}{ccc}
(n-2)\left(n_{1}-1\right) & (n-3) m_{1}+2 r_{1} & (n-1) n_{2} \\
(n-3) n_{1}+4 & n\left(m_{1}-1\right)-4\left(m_{1}-r_{1}\right) & (n-2) n_{2} \\
(n-1) n_{1} & (n-2) m_{1} & (n-2)\left(n_{2}-1\right)+r_{2}
\end{array}\right) .
$$

Thus we determine the VDC spectrum of $G_{1} \dot{\vee} G_{2}$.

\section{The VDC spectrum of $G_{1} \underline{\bigvee} G_{2}$}

In this section we present the VDC spectrum of $G_{1} \underline{\bigvee} G_{2}$.

Theorem 3. Let $G_{i}$ be $r_{i}$ regular graph with $n_{i}$ vertices and $m_{i}$ edges, for $i=1,2$. If $\left\{\lambda_{i 1}, \lambda_{i 2}, \ldots, \lambda_{i n_{i}}\right\}$ denotes the adjacency spectrum corresponding to the adjacency matrix $A_{i}$ of $G_{i}$, then, the $\operatorname{spec}_{V D C}\left(G_{1} \bigvee G_{2}\right)$ consists of

(i) $\lambda_{1 i}+3 \pm \sqrt{\left(\lambda_{1 i}+1\right)^{2}+4\left(\lambda_{1 i}+r_{1}\right)}-n$, for $i=2,3, \ldots, n_{1}$;

(ii) $-n+2$, repeated $m_{1}-n_{1}$ times;

(iii) $\lambda_{2 i}-n+2$, for $i=2,3, \ldots, n_{2}$;

(iv) the 3 roots of the equation

$$
\begin{gathered}
x^{3}-\left(n_{1} n-4 n_{1}+n_{2} n-2 n_{2}+m_{1} n-2 m_{1}+2 r_{1}+r_{2}-3 n+8\right) x^{2} \\
-\left(2 n_{1} n_{2} n-4 n_{1} n_{2}+n_{1} m_{1}+2 n_{1} r_{1} n-6 n_{1} r_{1}-n_{1} r_{2} n+4 n_{1} r_{2}+2 n_{1} n^{2}-12 n_{1} n+16 n_{1}\right. \\
+2 n_{2} m_{1} n-3 n_{2} m_{1}-2 n_{2} r_{1} n+4 n_{2} r_{1}+2 n_{2} n^{2}-10 n_{2} n+12 n_{2}-2 m_{1} r_{1} n+4 m_{1} r_{1}+2 m_{1} n^{2} \\
\left.-6 m_{1} n-m_{1} r_{2} n-2 r_{1} r_{2}+4 r_{1} n+2 m_{1} r_{2}+2 r_{2} n-6 r_{2}-3 n^{2}+16 n-20\right) x \\
-\left(2 n_{1} n_{2} m_{1}+4 n_{1} n_{2} r_{1} n-8 n_{1} n_{2} r_{1}+8 n_{1} n_{2}+2 n_{1} r_{1} n^{2}-8 n_{1} r_{2}-16 n_{1}\right. \\
-4 n_{2} m_{1} r_{1} n+6 n_{2} m_{1} r_{1}-3 n_{2} m_{1} n-16 n_{2}+2 m_{1} r_{1} r_{2} n-4 m_{1} r_{1} r_{2}-2 m_{1} r_{1} n^{2} \\
+8 m_{1} r_{1} n-8 m_{1} r_{1}-4 r_{1} r_{2}+2 n_{1} n_{2} n^{2}-8 n_{1} n_{2} n-n_{1} m_{1} r_{2}+n_{1} m_{1} n-2 n_{1} m_{1} \\
-2 n_{1} r_{1} r_{2} n+6 n_{1} r_{1} r_{2}+2 n_{1} r_{1} n^{2}-10 n_{1} r_{1} n+12 n_{1} r_{1}-n_{1} r_{2} n^{2}+6 n_{1} r_{2} n \\
+n_{1} n^{3}-8 n_{1} n^{2}+20 n_{1} n+2 n_{2} m_{1} n^{2}-4 n_{2} m_{1}-2 n_{2} r_{1} n^{2}+8 n_{2} r_{1}+n_{2} n^{3} \\
-8 n_{2} n^{2}+20 n_{2} n-m_{1} r_{2} n^{2}+2 m_{1} r_{2} n+4 m_{1} r_{2}+m_{1} n^{3}-4 m_{1} n^{2}+8 m_{1}-2 r_{1} r_{2} n \\
\left.+2 r_{1} n^{2}-8 r_{1}+r_{2} n^{2}-6 r_{2} n-n^{3}+8 n^{2}-20 n+8 r_{2}+16\right)=0 .
\end{gathered}
$$

where $n=n_{1}+m_{1}+n_{2}$. 
P r o o f. Given that $G_{1}$ and $G_{2}$ are regular graphs with regularity $r_{1}$ and $r_{2}$ respectively. Let $R$ be the incidence matrix of $G_{1} . G_{1} \bigvee G_{2}$ has $n=n_{1}+m_{1}+n_{2}$ vertices. With the proper labeling of vertices, the VDC matrix of $G_{1} \underline{V} G_{2}$ of order $n$ is given by

$$
C=\left(\begin{array}{ccc}
(n-4)(J-I)+2 A_{1} & (n-3) J+2 R & (n-2) J \\
(n-3) J+2 R^{T} & (n-2)(J-I) & (n-1) J \\
(n-2) J & (n-1) J & (n-2)(J-I)+A_{2}
\end{array}\right) .
$$

Let $\lambda_{1 i} \neq r_{1}$ be an eigenvalue of $A_{1}$ with an eigenvector $X$. By Perron-Frobenius theorem, $X$ is orthogonal to the all-one vector $J$.

Let us test the condition under which

$$
\Upsilon=\left(\begin{array}{c}
t X \\
R^{T} X \\
0
\end{array}\right)
$$

is an eigenvector of the given VDC matrix.

If $\Upsilon$ is an eigenvector of the VDC matrix of $G_{1} \underline{\bigvee} G_{2}$ corresponding to the eigenvalue $\eta$, then $C \Upsilon=\eta \Upsilon$ implies

$$
\left(\begin{array}{ccc}
(n-4)(J-I)+2 A_{1} & (n-3) J+2 R & (n-2) J \\
(n-3) J+2 R^{T} & (n-2)(J-I) & (n-1) J \\
(n-2) J & (n-1) J & (n-2)(J-I)+A_{2}
\end{array}\right)\left(\begin{array}{c}
t X \\
R^{T} X \\
0
\end{array}\right)=\eta\left(\begin{array}{c}
t X \\
R^{T} X \\
0
\end{array}\right)
$$

i. e.,

$$
-(n-4) t+2 t \lambda_{1 i}+2 \lambda_{1 i}+2 r_{1}=\eta t
$$

and

$$
2 t-(n-2)=\eta
$$

Substituting the value of $\eta$ from equation (3.2) in equation (3.1), we get a quadratic equation in $t$ as

$$
t^{2}-\left(1+\lambda_{1 i}\right) t-\left(\lambda_{1 i}+r_{1}\right)=0
$$

Hence

$$
t=\frac{\left(1+\lambda_{1 i}\right) \pm \sqrt{\left(1+\lambda_{1 i}\right)^{2}+4\left(\lambda_{1 i}+r_{1}\right)}}{2} .
$$

Thus corresponding to each eigenvalue $\lambda_{1 i} \neq r_{1}$ of $A_{1}$, we get two VDC eigenvalues $\eta=2 t+2-n$ of $G_{1} \bigvee G_{2}$ and hence a total of $2\left(n_{1}-1\right)$ VDC eigenvalues are obtained.

Now, -2 is an eigenvalue of $A\left(L\left(G_{1}\right)\right)$ with multiplicity $m_{1}-n_{1}$. Let $Z$ be an eigenvector of $A\left(L\left(G_{1}\right)\right)$ with eigenvalue -2 . Then, by Lemma $2, R Z=0$.

However,

$$
\Omega=\left(\begin{array}{l}
0 \\
Z \\
0
\end{array}\right)
$$

is an eigenvector of the VDC matrix of $G_{1} \bigvee G_{2}$ corresponding to the eigenvalue $-n+2$.

Let $\lambda_{2 i} \neq r_{2}$ be an eigenvalue of $G_{2}$ with an eigenvector $W$. Then,

$$
\Psi=\left(\begin{array}{c}
0 \\
0 \\
W
\end{array}\right)
$$

is an eigenvector of the VDC matrix of $G_{1} \underline{\bigvee} G_{2}$ corresponding to the eigenvalue $\lambda_{2 i}-n+2$, for $i=2,3, \ldots n_{2}$. 
Thus, we have obtained $n_{1}+m_{1}+n_{2}-3$ eigenvalues.

Next, we will determine the remaining three eigenvalues. We note that all the eigenvectors constructed are orthogonal to

$$
\left(\begin{array}{l}
J \\
0 \\
0
\end{array}\right), \quad\left(\begin{array}{l}
0 \\
J \\
0
\end{array}\right), \text { and } \quad\left(\begin{array}{l}
0 \\
0 \\
J
\end{array}\right)
$$

The remaining three eigenvectors are spanned by these three vectors and is of the form

$$
\Theta=\left(\begin{array}{c}
\alpha J \\
\beta J \\
\gamma J
\end{array}\right)
$$

for some $(\alpha, \beta, \gamma) \neq(0,0,0)$. Thus, if $\rho$ is an eigenvalue of $\mathrm{C}$ with an eigenvector $\Theta$ then from $C \Theta=\rho \Theta$, we can see that the remaining three eigenvalues are obtained from the matrix

$$
\left(\begin{array}{ccc}
(n-4)\left(n_{1}-1\right)+2 r_{1} & (n-3) m_{1}+2 r_{1} & (n-2) n_{2} \\
(n-3) n_{1}+4 & (n-2)\left(m_{1}-1\right) & (n-1) n_{2} \\
(n-2) n_{1} & (n-1) m_{1} & (n-2)\left(n_{2}-1\right)+r_{2}
\end{array}\right)
$$

\section{Conclusion}

In this paper we have computed the Vertex Distance Complement Spectrum of Subdivision Vertex Join, $G_{1} \dot{\vee} G_{2}$, and Subdivision Edge Join, $G_{1} \bigvee G_{2}$ of regular graphs $G_{1}$ and $G_{2}$. The work can be extended to graphs with diameter greater than two, graphs that are not regular etc. It is worth exploring the nature of the spectrum of graphs with arbitrary subdivisions.

\section{REFERENCES}

1. Brouwer A.E., Haemers W.H. Spectra of Graphs. New York: Springer, 2011. 250 p. DOI: 10.1007/978-1-4614-1939-6

2. Collatz L. V., Sinogowitz U. Spektren endlicher grafen. In: Abh. Math. Semin. Univ. Hambg., 1957. Vol. 21, No. 1. P. 63-77. DOI: 10.1007/BF02941924 (in German)

3. Cvetković D. M., Doob M., Sachs H. Spectra of Graphs - Theory and Application. New York: Academic Press, 1980. 368 p.

4. Indulal G. Spectrum of two new joins of graphs and infinite families of integral graphs. Kragujevac J. Math., 2012. Vol. 36, No. 1. P. 133-139.

5. Indulal G., Gutman I., Vijayakumar A. On distance energy of graphs. MATCH Commun. Math. Comput. Chem., 2008. Vol. 60, No. 2. P. 461-472.

6. Indulal G., Scaria D. C., Liu X. The distance spectrum of the subdivision vertex join and subdivision edge join of two regular graphs. Discrete Math. Lett., 2019. Vol. 1. P. 36-41.

7. Janežič D., Miličević A., Nikolić S., Trinajstić N. Graph-Theoretical Matrices in Chemistry. Florida: CRC Press, 2015. 174 p.

8. Varghese R. P., Susha D. Vertex distance complement spectra of regular graphs and its line graphs. Int. J. Appl. Math. Anal. Appl., 2017. Vol. 12, No. 2. P. 221-231.

9. Varghese R. P., Susha D. Vertex distance complement spectra of some graphs. Ann. Pure Appl. Math., 2018. Vol. 16, No. 1. P. 69-80. DOI: 10.22457/apam.v16n1a9 